\title{
MRCPsych Part II examination: proposed Critical Review Paper
}

A new paper examining candidates' skills at critical appraisal of research literature is to be introduced into the MRCPsych Part II examination, in place of the Short Answer Question (SAQ) paper. The notes below outline the principal reasons for this change, and summarise the objectives of the new examination.

\section{Reasons for introducing the Critical Review Paper}

(a) The importance of developing critical appraisal skills and evidence-based practice Good clinical practice in psychiatry, as in other medical specialities, relies on the combination of sound clinical judgement and the appropriate application of research-based evidence. These factors form the basis of evidence-based practice, the importance of which is widely acknowledged by managers and policy-makers as well as clinicians. Increasingly, in their clinical work and as advocates for their services and patients, psychiatrists need to acquire skills and confidence in critical appraisal of research evidence, and how this can be applied to clinical work. Senior doctors must be able to determine the value of individual published scientific articles, not only in terms of the scientific validity of their results and conclusions, but their applicability to clinical practice. The skills involved are of permanent value to the practising psychiatrist.

Journal Clubs, which have long formed part of most local academic programmes, have gone some way to meet this need. However, the organisation of these requires alteration to address the issues which will be covered in the new examination. More attention needs to be paid to the literature on systematic critical appraisal of research evidence. Most Journal Clubs have continued to retain their traditional format, with presentations failing to incorporate the tools and insights from this recent literature. It is also necessary to select more critically the articles chosen in such exercises and assess these more stringently if Journal Clubs are to be effective in preparing candidates for the new part of the examination.

\section{(b) Limitations of the current MRCPsych examination}

The MRCPsych Part II examination at present consists of six parts. There are two multiple choice question (MCQ) papers concerned with basic sciences and clinical topics, an essay, a Short Answer Question (SAQ) paper and the Clinical Examination, comprising Individual Patient Assessment (IPA) and Patient Management Problems (PMP) parts. In addition, there has for many years been a Research Option in the examinations, to encourage trainees to undertake and develop research. However, this option has only rarely been exercised.

An important requirement of the examination is that its different components should as far as possible measure different qualities and attributes of the candidates. This can be tested by measuring the extent of the correlations between the marks of candidates in the different parts of the examination. On this basis, the MCQ exams, the essay and the Clinical Examination appear to test different aspects of candidates' knowledge and experience. However, the SAQ paper marks are quite closely correlated with the MCQ (Clinical Topics paper) marks, and this paper does not introduce any extra dimension in assessment. Furthermore, the pass mark in the SAQ examination is difficult to set accurately. This is not a peerreferenced mark, but is set by the membership of the Examinations Sub-Committee when they assess the SAQ paper. Those involved in this exercise recognise that there is not a precise science in carrying out this task.

The Court of Electors, on the advice of the Examinations Sub-Committee, has recommended that the Research Option be discontinued after one year's grace period at the end of 1997. An alternative is needed to test the skills necessary to evaluate research and supplement the present examination curriculum. The SAQ paper will therefore be replaced with a Critical Review Paper.

\section{Aims of the Critical Review Paper}

The proposed Critical Review Paper is intended to assess skills and expertise that are not assessed in other parts of the MRCPsych examination. These include: 
(1) The ability to examine critically a published scientific paper, to assess the validity of the scientific information presented and determine its clinical importance and relevance.

(2) The ability to describe logically and clearly the results of such critical appraisal and the processes involved.

(3) The capacity to suggest further experiments that would confirm and/or expand understanding in the field under investigation.

(4) The ability to place information derived from a piece of research into context in the light of current views and practice.

To achieve these aims, candidates will require:

(1) Basic knowledge of different types of research design, and an understanding of the strengths and weaknesses of each type.

(2) Knowledge of common statistical techniques and the conditions under which each is appropriately applied.

(3) Skills in the systematic appraisal of published papers, particularly of their methodologies and reported results.
(4) Skills in determining the extent to which results or conclusions of published research may be applicable to particular situations relating to individual clinical cases or to a clinical service.

\section{Introduction of the new paper}

A working party of the Examinations SubCommittee has been set up to oversee the development and implementation of the new paper. This working party is chaired by $\mathrm{Dr}$ Stephen Tyrer, and includes Professor Jan Scott. Dr Mike Harris, Dr Femi Oyebode, Dr Robin Jacobson and Dr Tom Sensky. The new paper will be introduced into the MRCPsych Part II examination in Spring 1989. A pilot study has already been undertaken of a Critical Review Paper, and the results of this, and a further pilot to be undertaken in 1997, will guide the development of the final paper for the examination. The working party aims to publish sample question papers and answers for the examination, together with a brief reading list, by October 1997 .

The CrTtical Review PaPer Working Party 